08,03

\title{
Определение параметров эффекта Яна-Теллера в примесных центрах из ультразвуковых экспериментов: приложение к кристаллу ZnSe: $\mathrm{Ni}^{2+}$
}

\author{
(C) М.Н. Сарычев ${ }^{1}$, И.В. Жевстовских ${ }^{1,2, \uparrow}$, Н.С. Аверкиев ${ }^{3}$, И.Б. Берсукер ${ }^{4}$, \\ В.В. Гудков ${ }^{1}$, В.Т. Суриков ${ }^{5}$ \\ ${ }^{1}$ Уральский фредеральный университет им. Б.Н. Ельцина, \\ Екатеринбург, Россия \\ ${ }^{2}$ Институт физики металлов им. М.Н. Михеева УрО РАН, \\ Екатеринбург, Россия \\ ${ }^{3}$ Физико-технический институт им. А.Ф. Иофффе РАН, \\ Санкт-Петербург, Россия \\ ${ }^{4}$ Institute for Theoretical Chemistry, The University of Texas at Austin, \\ Austin, Texas USA \\ ${ }^{5}$ Институт химии твердого тела УрО РАН, \\ Екатеринбург, Россия \\ ฯ E-mail: zhevstovskikh@imp.uran.ru
}

(Поступила в Редакцию 27 августа 2018 г.)

Разработанная ранее методология использования ультразвуковых исследований для определения параметров эффекта Яна-Теллера (ЭЯТ) в примесных центрах в кристаллах получила дальнейшее развитие в настоящей работе в применении к примесям $\mathrm{Ni}^{2+}$ в $\mathrm{ZnSe}: \mathrm{Ni}^{2+}$. Для всех нормальных мод, распространяющихся вдоль оси [110], измерены температурные зависимости поглощения ультразвука в диапазоне частот 25-165 MHz. При температуре около $13 \mathrm{~K}$ для всех этих мод наблюдаются пики поглощения, свидетельствующие о наличии локальных искажений как тригонального, так и тетрагонального типов. Следуя ранее разработанной методике, показано, что эти аномалии поглощения обусловлены проявлением релаксационных переходов между эквивалентными локальными искажениями, индуцированными ЭЯТ в центрах $\mathrm{Ni}^{2+}$. В тетраэдрическом окружении селена этот ион находится в трехкратно вырожденном основном электронном состоянии ${ }^{3} T_{1}$, где могут реализоваться три типа ЭЯТ. Данные ультразвуковых экспериментов свидетельствуют о реализации $T \otimes\left(e+t_{2}\right)$ задачи, в которой поверхность потенциальной энергии имеет четыре эквивалентных минимума тригонального типа, слегка деформированных в сторону тетрагональных седлообразных точек. Оценены тригональные вибронные константы, энергии стабилизации тригональных минимумов и энергии активации барьеров между ними.

Работа выполнена в рамках государственного задания № АААА-А18-118020190098-5 и при поддержке РФФИ (грант № 18-32-00432).

DOI: 10.21883/FTT.2019.02.47132.243

\section{1. Введение}

Почти все примесные центры в кристаллах подвержены эффекту Яна-Теллера (ЭЯТ) в основном или возбужденном состоянии. Недавние исследования полупроводниковых кристаллов, содержащих $3 d$-примеси с ЭЯТ, показали эффективность ультразвуковых экспериментов в получении существенной информации о структуре и свойствах таких центров [1-5]. В зависимости от направления распространения деформационной волны ультразвука через центр с ЭЯТ, эквивалентные минимумы его адиабатической поверхности потенциальной энергии (ППЭ) становятся неэквивалентными, что непосредственно сказывается на измеряемых температурной и частотной зависимостях поглощения и скорости ультразвука и проявляется в виде характерных аномалий. Это позволяет определить из ультразвукового эксперимента характер искажений в центрах с ЭЯТ и другие параметры ППЭ примесного центра [2-5].
В настоящей работе методика ультразвукового исследования примесей с ЭЯТ получила дальнейшее развитие в применении к центрам $\mathrm{Ni}^{2+}$ в полупроводниковом монокристалле $\mathrm{ZnSe}: \mathrm{Ni}^{2+}$. Полупроводники, легированные магнитными примесями, обладают специфическими магнитными, магнито-транспортными и оптическими свойствами [6]. Широкозонные полупроводники, содержащие $3 d$-ионы, являются активной средой для лазеров среднего инфракрасного диапазона $[7,8]$ и могут быть использованы в качестве фотоприемников видимого и микроволнового излучения. Несмотря на то что в последние десятилетия было выполнено много исследований таких систем, их потенциальная важность для использования в устройствах электроники и оптоэлектроники определяет дальнейший повышенный интерес к изучению их строения и свойств $[9,10]$.

Двухвалентный ион $\mathrm{Ni}^{2+}\left(3 d^{8}\right)$ в кристалле $\mathrm{ZnSe}$ является примесью замещения и окружен четырьмя ближайшими ионами селена в тетраэдрическом окружении. 
Основное состояние ${ }^{3} \mathrm{~F}$ свободного иона $\mathrm{Ni}^{2+}$ расщепляется в тетраэдрическом поле $T_{d}$ симметрии на два триплета и синглет с основным ${ }^{3} T_{1}$ термом. В результате ЭЯТ [11] ППЭ локального комплекса с $T_{d}$-симметрией, включающего примесь $\mathrm{Ni}^{2+}$ и окружающие его атомы селена, существенно усложняется, приобретая несколько эквивалентных минимумов, в которых тетраэдр искажен в разных по симметрии эквивалентных направлениях. ЭЯТ в центрах $\mathrm{Ni}^{2+}$ был обнаружен ранее в кристалле $\mathrm{ZnSe}: \mathrm{Ni}^{2+}$ в спектрах фотолюминесценции и оптического поглощения $[12,13]$ и в структуре бесфононной линии в $\mathrm{CdS}: \mathrm{Ni}^{2+}[14]$.

Развитый в работах [1-5] метод ультразвукового исследования ЯТ-центров применен в данной работе к кристаллу $\mathrm{ZnSe}: \mathrm{Ni}^{2+}$. В отличие от многих предыдущих исследований ЭЯТ в примесных центрах, в рассматриваемом случае ЭЯТ создает как тригональные, так и тетрагональные искажения центра. Наблюдаемые особенности в температурных зависимостях поглощения ультразвука и их интерпретация привели к заключению, что в этом случае реализуется задача $T \otimes\left(e+t_{2}\right)$ ЭЯТ, в которой ППЭ имеет четыре эквивалентных тригональных минимума с тетрагональными седлообразными точками, что позволило определить вибронные константы и энергии активации.

\section{2. Образцы}

Монокристаллы ZnSe:Ni были выращены методом Бриджмена из расплава в условиях избыточного давления инертного газа. Содержание примесей переходных элементов определялось методом атомно-эмиссионной спектрометрии с индуктивно связанной плазмой (спектрометр Optima 8000, PerkinElmer, USA). Концентрация никеля в исследованном образце была $n_{\mathrm{Ni}}=4.5 \cdot 10^{19} \mathrm{~cm}^{-3}$, кроме того, наблюдалось небольшое количество примесей ванадия $n_{\mathrm{V}}=1.3 \cdot 10^{18} \mathrm{~cm}^{-3}$ и железа $n_{\mathrm{Fe}}=5.7 \cdot 10^{17} \mathrm{~cm}^{-3}$, концентрация других переходных элементов (титана, хрома, марганца, кобальта, меди) оказалась ниже, чем $5 \cdot 10^{17} \mathrm{~cm}^{-3}$.

Образцы для ультразвуковых измерений имели форму параллелепипеда размером приблизительно $5 \times 7 \times 9 \mathrm{~mm}^{3}$. Измерения поглощения ультразвука были выполнены с помощью фазочувствительной установки [15] в интервале частот 25-165 MHz, при температуpax от 4 до $130 \mathrm{~K}$. Ультразвуковые волны возбуждались и регистрировались с помощью пьезопреобразователей из $\mathrm{LiNbO}_{3}$, которые прикреплялись к противоположным поверхностям образца. Направление распространения упругих волн было вдоль кристаллографической оси [110], что позволяло определить скорость продольной волны $v_{l}=\sqrt{C_{l} / \rho}$, связанной с упругим модулем $C_{l}=\left(C_{11}+C_{12}+2 C_{44}\right) / 2$, и скорости двух поперечных волн $v_{t 1}=\sqrt{C_{44} / \rho}$ (когда вектор поляризации направлен вдоль оси [100]) и $v_{t 2}=\sqrt{\left(C_{11}-C_{12}\right) / 2 \rho}$ (вектор поляризации направлен вдоль оси $[1 \overline{1} 0])$; здесь $\rho-$ плотность кристалла, $C_{11}, C_{12}$ и $C_{44}-$ компоненты тензора упругого модуля кубического кристалла. Коэффициент поглощения ультразвуковой волны $\alpha$ определялся мнимой частью соответствующей компоненты тензора упругого модуля $\alpha=\left(\omega / 2 v_{i}\right)\left(\operatorname{Im}\left\{C_{i}\right\} / \operatorname{Re}\left\{C_{i}\right\}\right)$, где $\omega-$ круговая частота ультразвука, обозначает тип упругой волны.

\section{3. Результаты эксперимента}

Температурные зависимости поглощения всех нормальных мод, распространяющихся в кубическом кристалле $\mathrm{ZnSe}: \mathrm{Ni}$ на частоте около $50 \mathrm{MHz}$, представлены на рис. 1. Данные приведены для релаксационного поглощения $\alpha_{r e l}=\alpha-\alpha_{b}$, которое получено вычитанием из общего поглощения $\alpha$ фонового $\alpha_{b}$, аппроксимированного для каждой моды полиномом второй степени. Поперечные упругие волны, поляризованные вдоль [100] или [11이 кристаллографических осей, создают искажения в кристалле различной симметрии, тригональные или тетрагональные, соответственно. Локальная колебательная мода тригонального типа проявляется в поглощении продольной и поперечной моды, связанной с упругим модулем $C_{44}$, а тетрагонального типа - в поглощении продольной моды и поперечной, определяемой упругим модулем $\left(C_{11}-C_{12}\right) / 2$. В кристалле $\mathrm{ZnSe}: \mathrm{Ni}$ максимумы поглощения при температуре около $13 \mathrm{~K}$ наблюдаются для всех мод (рис. 1), что свидетельствует о проявлении в кристалле $\mathrm{ZnSe}: \mathrm{Ni}$ как тригональных, так и тетрагональных искажений. Величина максимумов поглощения существенно различается для волн различной поляризации. Наибольшее значение наблюдается для модуля $C_{44}$, что приводит к заметному поглощению продольной моды, величина же пика для поперечной волны

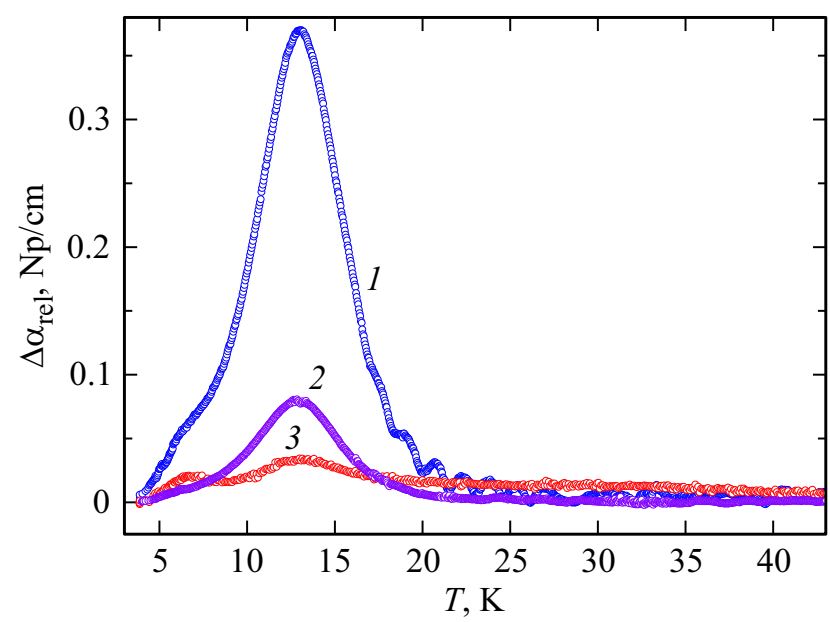

Рис. 1. Температурные зависимости релаксационного поглощения ультразвуковых волн в кристалле $\mathrm{ZnSe}: \mathrm{Ni}$, распространяющихся вдоль кристаллографической оси [110]. Кривая (1) соответствует упругому модулю $C_{44}$ (частота $54 \mathrm{MHz}$ ); $(2)-$ $\left(C_{11}+C_{12}+2 C_{44}\right) / 2$ (частота $\left.48 \mathrm{MHz}\right) ;(3)-\left(C_{11}-C_{12}\right) / 2$ (частота $56 \mathrm{MHz}$ ). $\Delta \alpha_{r e l}=\alpha_{r e l}(T)-\alpha_{r e l}\left(T_{0}\right), T_{0}=4 \mathrm{~K}$. 


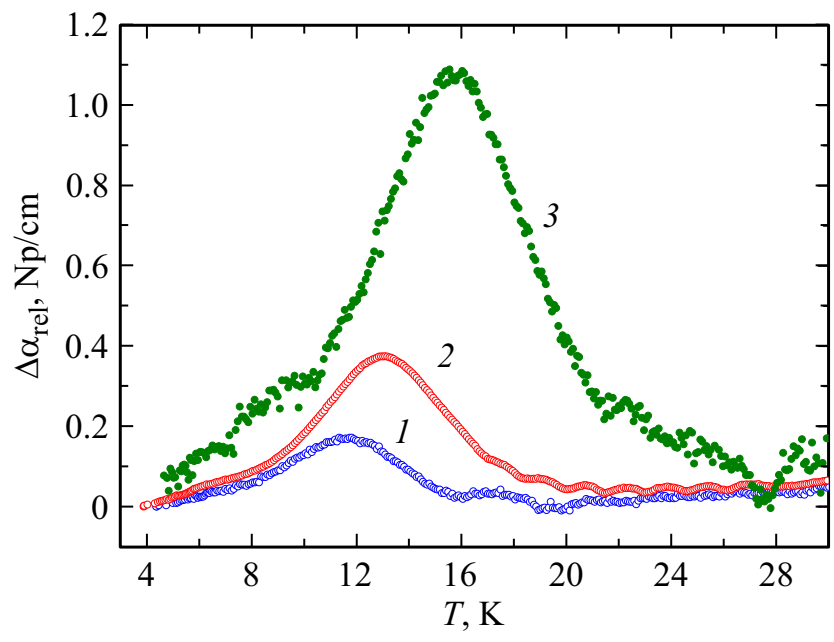

Рис. 2. Температурные зависимости релаксационного поглощения поперечных ультразвуковых волн в кристалле $\mathrm{ZnSe}: \mathrm{Ni}$, распространяющихся вдоль кристаллографической оси [110] с поляризацией вдоль $[100]$ (модуль $C_{44}$ ), измеренные на частотах $25 \mathrm{MHz}$ (кривая 1), $53 \mathrm{MHz}$ (2), $164 \mathrm{MHz}$ (3). $\Delta \alpha_{\text {rel }}=\alpha_{\text {rel }}(T)-\alpha_{\text {rel }}\left(T_{0}\right), T_{0}=4 \mathrm{~K}$.

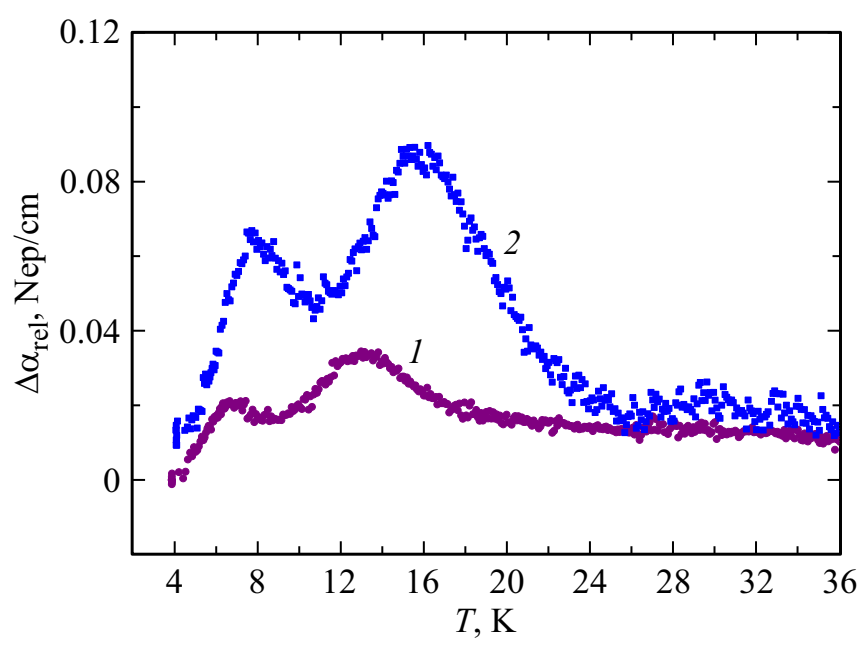

Рис. 3. Температурные зависимости релаксационного поглощения поперечных ультразвуковых волн в кристалле $\mathrm{ZnSe}: \mathrm{Ni}$, распространяющихся вдоль кристаллографической оси [110] с поляризацией вдоль [11̄0] (модуль $\left(C_{11}-C_{12}\right) / 2$ ), измеренные на частотах $56 \mathrm{MHz}$ (кривая 1), $167 \mathrm{MHz}$ (2). $\Delta \alpha_{r e l}=\alpha_{r e l}(T)-\alpha_{r e l}\left(T_{0}\right), T_{0}=4 \mathrm{~K}$.

с поляризацией вдоль [1필 оси (модуль $\left.\left(C_{11}-C_{12}\right) / 2\right)$ почти на порядок меньше. Ранее, нами были обнаружены в кристалле $\mathrm{ZnSe}: \mathrm{Ni}$ аномалии поглощения ультразвука для модулей $C_{l}=\left(C_{11}+C_{12}+2 C_{44}\right) / 2$ и $C_{44}$, и был сделан вывод о наличии лишь тригональных искажений $[16,17]$.

Релаксационная природа пиков поглощения подтверждается температурной зависимостью $\Delta \alpha_{\text {rel }}$, измеренной при различных фиксированных частотах (см. рис. 2, 3 и рис. 1 в работе [16] для продольных волн). С уве- личением частоты ультразвука положение пика сдвигается в более высокие температуры. Такое поведение релаксационного поглощения обязано множителю $f(\omega \tau)=\omega^{2} \tau /\left[1+(\omega \tau)^{2}\right]$, входящему в $\Delta \alpha_{r e l}(T)$ [10], где $\tau$ - время релаксации, и $\tau(T)$ определяется в основном переходами между искаженными конфигурациями ЯТ-центра (заметим, что в чистом кристалле $\mathrm{ZnSe}$ нет аномалий поглощения в температурной зависимости). Пик $\Delta \alpha_{\text {rel }}(T)$ наблюдается при условии $\omega \tau \approx 1$, при котором функция $f(\omega \tau)$ имеет максимум. При фиксированной частоте ультразвука $\omega$ изменение фактора $\omega \tau$ обусловлено температурной зависимостью времени релаксации. При больших значениях частоты $\omega$ условие $\omega \tau \approx 1$ реализуется в более высоких температурах, что и объясняет наблюдаемый сдвиг по температуре пика на зависимости $\Delta \alpha_{\text {rel }}(T)$.

Кроме пика поглощения вблизи $13 \mathrm{~K}$ для ультразвуковых волн различной поляризации, также был обнаружен небольшой максимум вблизи температуры $7 \mathrm{~K}$ для поперечной моды, связанной с упругим модулем $\left(C_{11}-C_{12}\right) / 2$ (рис. 1 и 3 ). В исследованном кристалле $\mathrm{ZnSe}: \mathrm{Ni}^{2+}$ кроме примеси никеля содержалось небольшое количество примесей ванадия и железа, что может также приводить к релаксационному поглощению ультразвука, так как ионы $\mathrm{V}^{2+}$ и $\mathrm{Fe}^{2+}$ в тетраэдрической координации являются активными ЯТ-центрами $[18,19]$. Но аномалии поглощения в кристалле $\mathrm{ZnSe}: \mathrm{V}^{2+}$ наблюдались при температурах ниже $4 \mathrm{~K}$ [18]. Что касается примеси железа, то пик поглощения в образце $\mathrm{ZnSe}: \mathrm{Fe}^{2+}$ для поперечной моды, связанной с упругим модулем $\left(C_{11}-C_{12}\right) / 2$, как раз наблюдался вблизи $7 \mathrm{~K}[19]$, поэтому проявление в образце $\mathrm{ZnSe}: \mathrm{Ni}^{2+}$ таких же аномалий поглощения вблизи $7 \mathrm{~K}$ мы относим к наличию примеси железа. Высота этого пика в данном кристалле на порядок меньше, чем в $\mathrm{ZnSe}: \mathrm{Fe}^{2+}[19]$, но и содержание примеси железа также значительно меньше, а его релаксационная природа подтверждается характерным сдвигом положения пика в более высокие температуры при повышении частоты (рис. 3).

\section{4. Обсуждение}

\section{1. Время релаксации}

Измеренная температурная зависимость поглощения ультразвука $\alpha(T)$ позволяет получить температурную зависимость времени релаксации $\tau(T)$, полагая, что пик на температурной зависимости поглощения обусловлен релаксацией в системе невзаимодействующих ян-теллеровских (ЯТ) ионов $\mathrm{Ni}^{2+}$ в кристалле $\mathrm{ZnSe}$. Вклад от других механизмов был аппроксимирован монотонной функцией $\alpha_{b}(T)$ (в данном случае - полиномом второй степени). Тогда выражение для $\tau(T)$ можно представить 


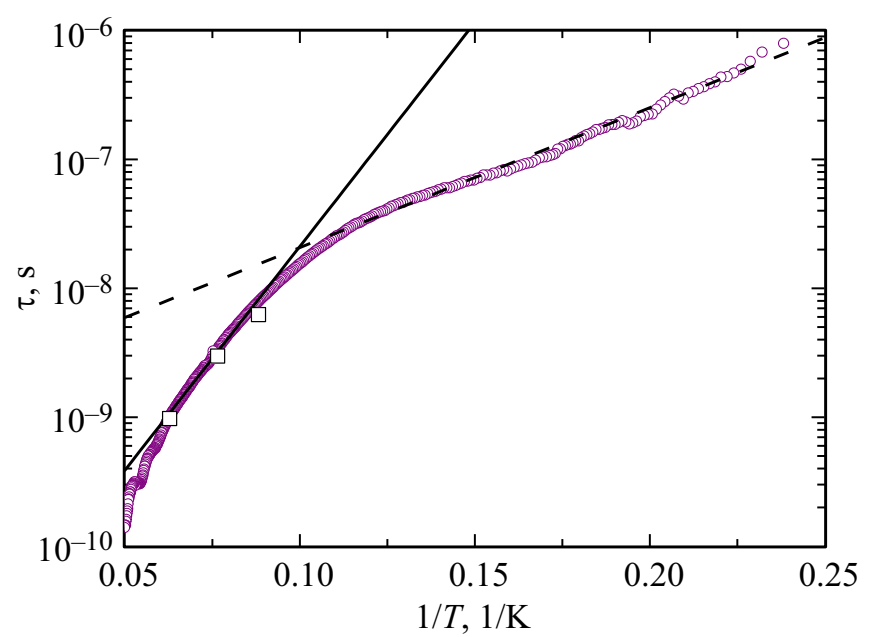

Рис. 4. Температурная зависимость времени релаксации, полученная из поглощения поперечной ультразвуковой волны, распространяющейся вдоль кристаллографической оси [110] с поляризацией вдоль [100] на частоте $54 \mathrm{MHz}$ (кружки). Квадраты показывают значения $\tau$, полученные из зависимостей $\Delta \alpha_{r e l}(T)$, измеренные при различных частотах. Штриховая линия - функция $\tau(1 / T)=\tau_{1} \exp \left(V_{1} / k_{B} T\right)$ c $\tau_{1}=1.7 \times 10^{-9} \mathrm{~s}, \quad V_{1}=25 \mathrm{~K}=17 \mathrm{~cm}^{-1} ;$ сплошная линия - функция $\tau(1 / T)=\tau_{2} \exp \left(V_{2} / k_{B} T\right)$ с $\tau_{2}=1.7 \times 10^{-12} \mathrm{~s}$, $V_{2}=80 \mathrm{~K}=56 \mathrm{~cm}^{-1}$.

в виде $[1,17]$

$$
\tau(T)=\frac{1}{\omega}\left[\frac{\alpha_{r e l}\left(T_{1}\right) T_{1}}{\alpha_{r e l}(T) T} \pm \sqrt{\left(\frac{\alpha_{r e l}\left(T_{1}\right) T_{1}}{\alpha_{r e l}(T) T}\right)^{2}-1}\right],
$$

где $T_{1}$ - температура, при которой выполняется условие $\omega \tau=1$ и которая может быть определена из положения максимума функции $\phi(T)=\alpha_{r e l}(T) \cdot T$. Функция $\tau(1 / T)$, полученная с помощью (1) для поперечной ультразвуковой волны, связанной с упругим модулем $C_{44}$, приведена на рис. 4. Белыми квадратами на рис. 4 показаны значения $\tau$, определенные из условия $\omega \tau=1$ и измерений поглощения на разных частотах (рис. 2). Видно достаточно хорошее совпадение значений времени релаксации, определенных разными способами. Полученная зависимость $\tau(1 / T)$ позволяет оценить энергию активации согласно уравнению Аррениуса $\tau(1 / T)=\tau_{0} \exp \left(V_{0} / k_{B} T\right)$, где $\tau_{0}^{-1}$ - частотный фактор, $V_{0}$ - энергия активации, $k_{B}$ - постоянная Больцмана. При температуре около $10 \mathrm{~K}$ происходит переход от низкотемпературного режима активации (с энергией $V_{1}=17 \mathrm{~cm}^{-1}$ при $T<10 \mathrm{~K}$ ) к высокотемпературному (с энергией $V_{2}=56 \mathrm{~cm}^{-1}$ при $T>10 \mathrm{~K})$.

Оптические исследования примесного центра $\mathrm{Ni}^{2+}$ в кристалле $\mathrm{ZnSe}$ выявили тонкую структуру оптических переходов с основного состояния ${ }^{3} T_{1}(F)$ центра, связанную с проявлением ЭЯТ и возникновением переходов на возбужденные вибронные уровни терма ${ }^{1} T_{2}[12,13]$. Положение ближайших сателлитных линий, наблюдаемых в спектре излучения в $\mathrm{ZnSe}: \mathrm{Ni}^{2+}$ было соответственно 40 и $64 \mathrm{~cm}^{-1}$ [13]. Полагая, что релаксация в ультразвуковом эксперименте определяется однофононными процессами через возбужденные состояния, можно считать, что энергия активации определяет энергию возбужденного вибронного состояния примесного центра $\mathrm{Ni}^{2+}$. Низкотемпературная энергия активации $V_{1}=17 \mathrm{~cm}^{-1}$ может быть связана с расщеплением основного состояния вследствие тригонального искажения центра в минимуме ППЭ. При повышении температуры мы переходим на следующий возбужденный уровень с энергией $V_{2}=56 \mathrm{~cm}^{-1}$. Разность между этими энергетическими состояниями $V_{2}-V_{1}=39 \mathrm{~cm}^{-1}$ хорошо согласуется со значением $40 \mathrm{~cm}^{-1}$, определенным в [13], как положение ближайшей сателлитной линии в спектре излучения в $\mathrm{ZnSe}: \mathrm{Ni}^{2+}$, связанной с расщеплением основного ${ }^{3} T_{1}(F)$-состояния.

\section{2. Линейная вибронная константа связи и энергия стабилизации в ЭЯТ}

Как уже упоминалось выше, основное электронное состояние иона $\mathrm{Ni}^{2+}$ в тетраэдрическом окружении в кубическом кристалле $\mathrm{ZnSe}{ }^{3} T_{1}$ трехкратно вырождено. Наш эксперимент выявил наличие как тригональных, так и тетрагональных локальных искажений кластера $\left[\mathrm{NiSe}_{4}\right]$, что свидетельствует о проявлении $T \otimes\left(e+t_{2}\right)$ задачи ЭЯТ [11]. В этом случае ППЭ зависит от пяти координат, двух тетрагонального $e$-типа $-Q_{\varepsilon}$ и $Q_{\theta}$, и трех тригонального $t_{2}$-типа $-Q_{\xi}, Q_{\eta}$ и $Q_{\xi}$. В линейном приближении оператор потенциальной энергии $\hat{U}$ вибронного Гамильтониана содержит только две вибронные константы связи: $F_{E}$ для тетрагональных и $F_{T}$ для тригональных смещений [11]

$\hat{U}=\left\|\begin{array}{ccc}F_{E}\left(\frac{1}{2} Q_{\theta}-\frac{\sqrt{3}}{2} Q_{\varepsilon}\right) & -F_{T} Q_{\xi} & -F_{T} Q_{\eta} \\ -F_{T} Q_{\xi} & F_{E}\left(\frac{1}{2} Q_{\theta}+\frac{\sqrt{3}}{2} Q_{\varepsilon}\right) & -F_{T} Q_{\xi} \\ -F_{T} Q_{\eta} & -F_{T} Q_{\xi} & -F_{T} Q_{\xi}\end{array}\right\|$.

Корни секулярного уравнения $\quad\left\|\hat{U}_{\gamma \gamma^{\prime}}-\varepsilon_{k}\right\|=0$, $\left(\gamma, \gamma^{\prime}=1,2,3\right)$ дают электронные энергии $\varepsilon_{k}(Q)$ $(k=1,2,3)$, что в комбинации с упругой энергией описывает три ветви ППЭ в пятимерном пространстве координат $Q$ следующим образом [20]:

$$
E_{k}(Q)=\frac{1}{2} K_{E}\left(Q_{\varepsilon}^{2}+Q_{\theta}^{2}\right)+\frac{1}{2} K_{T}\left(Q_{\xi}^{2}+Q_{\eta}^{2}+Q_{\xi}^{2}\right)+\varepsilon_{k}(Q),
$$

где $K_{E}$ и $K_{T}$ - исходные силовые константы (силовые константы без учета вибронной связи). Исследование такой поверхности в общем виде затруднительно, но в зависимости от соотношения между линейными вибронными константами $F_{E}$ и $F_{T}$ адиабатический потенциал имеет либо тетрагональные, либо тригональные глобальные минимумы, а также орторомбические в 
более высоких порядках [21], определяя соответствующие искажения тетраэдрического окружения ЯТ-иона. В наших экспериментах ЯТ искажения, определяемые модулем тригонального типа $C_{44}$, значительно (почти на порядок) превосходят искажения тетрагонального типа, связанные с модулем $\left(C_{11}-C_{12}\right) / 2$. Тригональный характер искажений в кристалле $\mathrm{ZnSe}: \mathrm{Ni}^{2+}$ также был выявлен при исследовании тонкой структуры спектров оптического поглощения [12]. Так как тетрагональные искажения вносят значительно меньший вклад, ППЭ в этой $T \otimes\left(e+t_{2}\right)$ задаче имеет четыре эквивалентных минимума тригонального типа с тремя тетрагональными седлообразными точками [11]. Энергия стабилизации центра ЭЯТ в этом случае определяется формулой

$$
E_{\mathrm{JT}}=\frac{2 F_{T}^{2}}{3 K_{T}} .
$$

Линейную вибронную константу связи $F_{T}$ можно оценить из данных ультразвукового эксперимента, используя метод, развитый в работах [2-5]. Для изотермического вклада ЯТ-центров в полный упругий модуль $C_{\mathrm{JT}}^{T}$ можно записать следующее выражение [5]:

$$
C_{\mathrm{JT}}^{T}=\left(\frac{\partial^{2} A_{\mathrm{JT}}}{\partial \varepsilon^{2}}\right)_{\varepsilon=0}=-n k_{B} T\left(\frac{\partial^{2} \ln Z}{\partial \varepsilon^{2}}\right)_{\varepsilon=0},
$$

где $A_{\mathrm{JT}}-$ свободная энергия Гельмгольца ЯТ-центров, $n$ - концентрация ЯТ-центров, $\varepsilon-$ деформация, производимая звуковой волной, $Z-$ статистическая сумма состояний, которая для четырех минимумов тригонального типа в нашей задаче имеет вид

$$
Z=\sum_{i=1}^{4} \exp \left(-E_{i} / k_{B} T\right)
$$

где $E_{i}(\varepsilon)$ - энергия $i$-конфигурации в поле деформаций. Релаксационное поглощение ультразвуковой волны в предположении, что вклад ЯТ-центров в адиабатический модуль равен нулю, то есть $C_{\mathrm{JT}}^{S}=0$, определяется выражением [5]

$$
\frac{\alpha_{r e l}}{k_{0}}=-\frac{C_{\mathrm{JT}}^{T}}{2 C_{0}} \frac{\omega \tau}{1+(\omega \tau)^{2}},
$$

где $k_{0}=\omega / v_{0}-$ волновой вектор ультразвука, $C_{0}-$ упругий модуль $C_{44}$ в чистом $\mathrm{ZnSe}$. Так как максимум релаксационного поглощения наблюдается при условии $\omega \tau\left(T_{1}\right)=1$, то (7) с учетом (5) принимает вид

$$
\alpha_{r e l}\left(T_{1}\right)=-\frac{k_{0} C_{\mathrm{JT}}^{T}}{4 C_{0}}=n k_{B} T \frac{k_{0}}{4 C_{0}}\left(\frac{\partial^{2} \ln Z}{\partial \varepsilon^{2}}\right)_{\varepsilon=0} .
$$

Для упругого модуля $C_{44}$ в кубическом кристалле, когда направление волнового вектора ультразвука направлено вдоль кристаллографической оси [110], а вектор поляризации вдоль оси [100], отличны от нуля компоненты тензора деформаций $\varepsilon_{i j} \varepsilon_{y z}=\varepsilon_{x z} \equiv \varepsilon_{4}$, и для энергий четырех тригональных минимумов $E_{i}$ в (6), получаем

$$
E_{1}=E_{2}=0, \quad E_{3}=d \varepsilon_{4}, \quad E_{4}=-d \varepsilon_{4},
$$

где $d-$ константа деформационного потенциала. С этими данными (6) примет вид

$$
Z=2+\exp \left(\frac{d \varepsilon_{4}}{k_{B} T}\right)+\left(-\frac{d \varepsilon_{4}}{k_{B} T}\right) .
$$

Значение второй производной $\ln Z$ по деформации $\varepsilon_{4}$ в пределе $\varepsilon_{4} \rightarrow 0$ равно

$$
\begin{aligned}
\left(\frac{\partial^{2} \ln Z}{\partial \varepsilon_{4}^{2}}\right)_{\varepsilon_{4} \rightarrow 0} & =\left(-\frac{1}{Z^{2}}\left(\frac{\partial Z}{\partial \varepsilon_{4}}\right)^{2}+\frac{1}{Z}\left(\frac{\partial^{2} Z}{\partial \varepsilon_{4}^{2}}\right)\right)_{\varepsilon_{4} \rightarrow 0} \\
& =\frac{d^{2}}{2 k_{B}^{2} T^{2}} .
\end{aligned}
$$

Подставляя его в $(8)$, получим выражение для $\alpha_{r e l}$

$$
\alpha_{r e l}\left(T_{1}\right)=\frac{k_{0} n d^{2}}{8 C_{0} k_{B} T} .
$$

Для расчета линейной вибронной константы $F_{T}$ полагаем, что $F_{T}=d / a_{0}$, где $a_{0}-$ расстояние между ионом никеля и ближайшим ионом селена. Тогда из (12) следует

$$
F_{T}^{2}=\frac{8 C_{0} k_{B} T_{1} \alpha_{r e l}\left(T_{1}\right)}{n k_{0} a_{0}^{2}},
$$

где $k_{0}=\omega / v_{0}$, где $v_{0}=\left(C_{0} / \rho\right)^{0.5}$. Расчет $F_{T}$ был выполнен с использованием следующих значений параметров: $C_{0}=4.06 \cdot 10^{11} \mathrm{dyn} / \mathrm{cm}^{2} \quad[22], \quad a_{0}=2.46 \AA$, $\rho=5.42 \mathrm{~g} / \mathrm{cm}^{3}$, значение $T_{1} \alpha_{r e l}\left(T_{1}\right)$ взято из экспериментальной зависимости поглощения для модуля $C_{44}$ на частоте $54 \mathrm{MHz}$. Для концентрации ЯТ-центров было взято значение $n=2.3 \cdot 10^{19} \mathrm{~cm}^{-3}$ немного меньшее, чем было определено методом атомно-эмиссионной спектроскопии, так как не все ионы никеля являются примесями замещения и оказываются в тетраэдрическом окружении. Таким образом, было получено $\left|F_{T}\right|=1.31 \cdot 10^{-5} \mathrm{dyn}=7.1 \mathrm{eV} / \AA$.

Оценка первичной силовой константы $K_{T}$ (силовой константы взаимодействия иона $\mathrm{Ni}^{2+}$ при его смещении в тригональном направлении в тетраэдрическом окружением селена без учета ЭЯТ) более сложна, потому что она отражается в экспериментальных данных только косвенно. Формально $K_{T}=\omega_{\tau}^{2} \cdot M$, где $\omega_{\tau}-$ частота соответствующего локального колебания, а $M$ приведенная масса колеблющейся системы, но значение $\omega_{\tau}$ неизвестно. Можно полагать, что наименьшая частота поперечных акустических фононов чистого кристалла $\mathrm{ZnSe} \hbar \omega \sim 60 \mathrm{~cm}^{-1}[23,24]$ приблизительно отражает частоту соответствующего колебания иона $\mathrm{Zn}^{2+}$ в окружении селена, но для иона $\mathrm{Ni}^{2+}$ с меньшим ионным радиусом в том же окружении следует ожидать меньшую частоту. Более приемлемую оценку можно получить из оптических данных по этому кристаллу [12], из которых следует, что энергия стабилизации ${ }^{1} T_{2}$ терма ЭЯТ равна $E_{\mathrm{JT}}^{T}=81 \mathrm{~cm}^{-1}$. Если пренебречь возможной небольшой разницей в этой величине для ${ }^{3} T_{1}$ терма, мы получаем из уравнения (4), 
что $K_{T}=0.53 \cdot 10^{4} \mathrm{dyn} / \mathrm{cm}$ и $\hbar \omega=43 \mathrm{~cm}^{-1}$. Значение энергии стабилизации $E_{\mathrm{JT}}^{T}=81 \mathrm{~cm}^{-1}$ приблизительно согласуется с высотой барьера между минимумами, оцененного по энергии активации, полученной из ультразвукового эксперимента. Действительно, высокотемпературная (выше $10 \mathrm{~K}$ ) энергия активации равна $56 \mathrm{~cm}^{-1}$, и если к ней добавить энергию нулевых колебаний $\hbar \omega_{\tau} / 2=22 \mathrm{~cm}^{-1}$, мы получаем $E_{\mathrm{JT}}^{T}=78 \mathrm{~cm}^{-1}$.

\section{5. Заключение}

Ультразвуковые методы исследования строения и свойств примесных центров в кристаллах, подверженных ЭЯТ, получили дальнейшее экспериментальное и теоретическое обоснование на примере кристалла $\mathrm{ZnSe}: \mathrm{Ni}^{2+}$. В отличие от многих предыдущих работ, аномалии в поглощении ультразвука и их зависимости от направления распространения волны в этом кристалле свидетельствуют о наличии в нем как тетрагональных, так и тригональных искажений, индуцированных ЭЯТ в примесных центрах $\mathrm{Ni}^{2+}$. Это позволило сделать вывод о том, что в данном случае реализуется $T \otimes\left(e+t_{2}\right)$ задача ЭЯТ, в которой поверхность потенциальной энергии имеет четыре эквивалентных минимума тригонального типа, каждый из которых слегка деформирован тетрагонально в направлении тетрагональных седлообразных точек. В рамках линейного приближения теории ЭЯТ получены численные значения тригональной вибронной константы и значения энергии стабилизации тригональных минимумов. Реконструирована температурная зависимость времени релаксации между эквивалентными ян-теллеровскими искажениями тригонального типа, из которой были определены нижайшие колебательные уровни центра $\mathrm{Ni}^{2+}$ в предположении, что релаксация определяется однофононными процессами через возбужденные состояния.

\section{Список литературы}

[1] V.V. Gudkov. In: The Jahn-Teller effect / Ed. H. Koppel, D.R. Yarkony, H. Barentzen. Springer, Heidelberg (2009).

[2] V.V. Gudkov, I.B. Bersuker, I.V. Zhevstovskikh, Yu.V. Korostelin, A.I. Landman. J. Phys.: Condens. Matter 23, 115401 (2011).

[3] N.S. Averkiev, I.B. Bersuker, V.V. Gudkov, K.A. Baryshnikov, G.V. Colibaba, I.V. Zhevstovskikh, V.Yu. Mayakin, A.M. Monakhov, D.D. Nedeoglo, M.N. Sarychev, V.T. Surikov. Phys. Status Solidi B 251, 1590 (2014).

[4] I.V. Zhevstovskikh, I.B. Bersuker, V.V. Gudkov, N.S. Averkiev, M.N. Sarychev, S. Zherlitsyn, S. Yasin, G.S. Shakurov, V.A. Ulanov, V.T. Surikov. J. Appl. Phys. 119, 225108 (2016).

[5] N.S. Averkiev, I.B. Bersuker, V.V. Gudkov, I.V. Zhevstovskikh, M.N. Sarychev, S. Zherlitsyn, S. Yasin, G.S. Shakurov, V.A. Ulanov, V.T. Surikov. J. Phys. Soc. Jpn. 86, 114604 (2017).

[6] K.A. Kikoin, V.N. Flerov. Transition Metal Impurities in Semiconductors: Electronic Structure and Physical Properties. World Scientific, Singapore. (1994).
[7] V.V. Fedorov, S.B. Mirov, A. Gallian, D.V. Badikov, M.P. Frolov, Yu.V. Korostelin, V.I. Kozlovsky, A.I. Landman, Yu.P. Podmar'kov, V.A. Akimov, A.A. Voronov. IEEE J. Quant. Electron. 42, 9, 907 (2006).

[8] I.T. Sorokina, E. Sorokin, S.B. Mirov, V.V. Fedorov, V. Badikov, V. Panyutin, K. Schaffers. Opt. Lett. 27, 1040 (2002).

[9] Ю.А. Ницук, Ю.Ф. Ваксман, В.В. Яцун. ФТП 46, 10, 1288 (2012).

[10] V. Sirkeli, I. Radevici, K. Sushkevich, H. Huhtinen, N. Nedeoglo, D. Nedeoglo, P. Paturi. Solid State Sci. 50, 74 (2015).

[11] I.B. Bersuker. The Jahn-Teller Effect. Cambridge University Press, Cambridge. (2006).

[12] J.M. Baranovski, J.M. Noras, J.W. Allen. J. Phys. C 7, 4529 (1974).

[13] A. Karipidou, H. Nelkowski, G. Roussos. J. Crystal Growth 59, 307 (1982).

[14] Ю.П. Гнатенко, А.Х. Рожко. Письма в ЖЭТФ 24, 3, 125 (1976).

[15] V.V. Gudkov, J.D. Gavenda. Magnetoacoustic polarization phenomena in solids. Springer-Verlag, N. Y. etc. (2000).

[16] В.В. Гудков, А.Т. Лончаков, В.И. Соколов, И.В. Жевстовских, Н.Б. Груздев. ФТТ 47, 8, 1498 (2005).

[17] V. Gudkov, A. Lonchakov, V. Sokolov, I. Zhevstovskikh, N. Gruzdev. Phys. Status Solidi B 242, 3, R30 (2005).

[18] В.В. Гудков, А.Т. Лончаков, В.И. Соколов, И.В. Жевстовских, В.Т. Суриков. ФТТ 50, 9, 1703 (2008).

[19] В.В. Гудков, А.Т. Лончаков, И.В. Жевстовских, В.И. Соколов, В.Т. Суриков. ФНТ 35, 1, 99 (2009).

[20] U. Opik, M.H.L. Pryce. Proc. R. Soc. London A 238, 425 (1957).

[21] И.Б. Берсукер, В.З. Полингер. ЖЭТФ, 66, 2078 (1974).

[22] B.H. Lee. J. Appl. Phys. 41, 2984 (1970).

[23] D.N. Talwar, M. Vandevyver, K. Kunc, M. Zigone. Phys. Rev. B 24, 741 (1981).

[24] R.K. Kremer, M. Cardona, R. Lauck, G. Siegle, A.H. Romero. Phys. Rev. B 85, 035208 (2012).

Редактор Ю.Э. Китаев 